\title{
On the Statistics of Spectrogram Reassignment Vectors*
}

\author{
ERIC CHASSANDE-MOTTIN \\ echassan@physique.ens-lyon.fr \\ PATRICK FLANDRIN \\ flandrin@physique.ens-lyon.fr \\ Laboratoire de Physique, Ecole Normale Supérieure de Lyon, 46 allée d'Italie, \\ 69364 Lyon Cedex 07, France \\ FRANÇOIS AUGER \\ auger@ge44.univ-nantes.fr \\ GE44, CRTT, Bd de l'Université, BP 406, 44602 Saint-Nazaire Cedex, France \\ Received August 20, 1996; Revised February 8, 1997 \\ Abstract. Reassignment is a non-linear technique which can improve on the localization of a spectrogram by \\ moving its values according to a suitable vector field. Statistical properties of spectrogram reassignment vectors \\ are investigated in detail. Closed form expressions are given when the observation consists in a non-random \\ component embedded in white Gaussian circular noise, and when the analysis window is Gaussian. An extension \\ to arbitrary windows is also proposed and theoretical claims are supported by numerical simulations.
}

Keywords: time-frequency distributions, reassignment methods

\section{Introduction}

Reassignment is a non-linear technique which has recently been reintroduced and put forward as an efficient means of getting sharply localized time-frequency distributions [1]. The technique consists in moving the value of a time-frequency distribution from the point where it has been computed to a new location which is more representative of the local signal energy distribution. Reassignment is based on a vector field which conveys a lot of information about the signal structure and it is therefore an important issue to quantify properties of this vector field, especially from a statistical point of view. This paper is devoted to first theoretical investigations of this question which - up to now - has received no attention in the literature, although it is believed to be of a key importance for many further signal processing applications based on reassigned distributions. While reassignment is a very general principle which can be applied to almost any time-frequency distribution, discussion will be restricted here to spectrograms only. Let us finally note that, due to space limitations, most proofs of the results presented here have been omitted, but they can be found in [2].

\section{Basics and assumptions}

In order to reassign the spectrogram associated to the short-time Fourier transform (STFT)

* This work was supported in part by the GdR 134 CNRS “Traitement du Signal et Images." 


$$
F_{x}^{(h)}(t, \nu)=\int_{-\infty}^{+\infty} x(t-\tau) h^{*}(\tau) e^{2 \pi \imath \nu \tau} d \tau
$$

based on a window $h(t)$, it is necessary to introduce two auxiliary STFT's based on the windows $(\mathcal{T} h)(t)=t h(t)$ and $(\mathcal{D} h)(t)=(d / d t) h(t)$. Given these three STFT's, reassignment amounts to shift the value of $\left|F_{x}^{(h)}(t, \nu)\right|^{2}$ from $(t, \nu)$ to a new location $(\hat{t}(t, \nu), \hat{\nu}(t, \nu))$ characterized by [1]

$$
(\hat{t}(t, \nu), \hat{\nu}(t, \nu))=\left(t-\operatorname{Re}\left\{\frac{F_{x}^{(\mathcal{T} h)}(t, \nu)}{F_{x}^{(h)}(t, \nu)}\right\}, \nu+\frac{1}{2 \pi} \operatorname{Im}\left\{\frac{F_{x}^{(\mathcal{D} h)}(t, \nu)}{F_{x}^{(h)}(t, \nu)}\right\}\right) .
$$

Identifying the time-frequency plane with the complex plane, it is therefore possible to define at each time-frequency point a dimensionless reassignment vector $r(t, \nu)$ according to

$$
r(t, \nu)=\frac{\hat{t}(t, \nu)-t}{\Delta t_{h}}+\imath \frac{\hat{\nu}(t, \nu)-\nu}{\Delta \nu_{h}},
$$

where $\Delta t_{h}$ and $\Delta \nu_{h}$ stand, respectively, for the mean-square duration and bandwidth of the analysis window. This study is devoted to statistical properties of $r(t, \nu)$ in the case of observations corrupted by a zero-mean noise $n(t)$ assumed to be white, Gaussian and analytic (hence, circular [3]), i.e., such that

$\mathbf{E}[\operatorname{Re}\{n(t)\} \operatorname{Re}\{n(s)\}]=\mathbf{E}[\operatorname{Im}\{n(t)\} \operatorname{Im}\{n(s)\}]=\frac{\sigma^{2}}{2} \delta(t-s) ; \mathbf{E}[n(t) n(s)]=0$

for any $(t, s) \in \mathbf{R}^{2}$, with its real and imaginary parts a Hilbert transform pair.

\section{Statistics of reassignment vectors for Gabor spectrograms}

Let us first consider a Gaussian window $h(t)=2^{1 / 4} \lambda^{-1 / 2} \exp \left(-\pi(t / \lambda)^{2}\right)$, a situation referred to as a Gabor spectrogram. In this case, we have $\Delta t_{h}=(4 \pi)^{-1 / 2} \lambda$ and $\Delta \nu_{h}=$ $(4 \pi)^{-1 / 2} / \lambda$, and $(\mathcal{T} h)(t)=t h(t)$ and $(\mathcal{D} h)(t)=(d / d t) h(t)$ happen to be proportional to each other. The relative reassignment vector reduces to

$$
r(t, \nu)=-\frac{\sqrt{4 \pi}}{\lambda} \frac{F_{2}}{F_{1}}
$$

where $F_{1}$ and $F_{2}$ are simplified notations for $F_{x}^{(h)}(t, \nu)$ and $F_{x}^{(\mathcal{T} h)}(t, \nu)$, respectively.

\subsection{The "noise only" case}

In the "noise only" case where the observation is $x(t)=n(t)$, it follows from properties related to both linear filtering and analytic circularity that the vector $\underline{F}=\left[F_{1} F_{2}\right]^{t}$ is zero-mean and Gaussian circular, with a joint probability density function (pdf) given by 

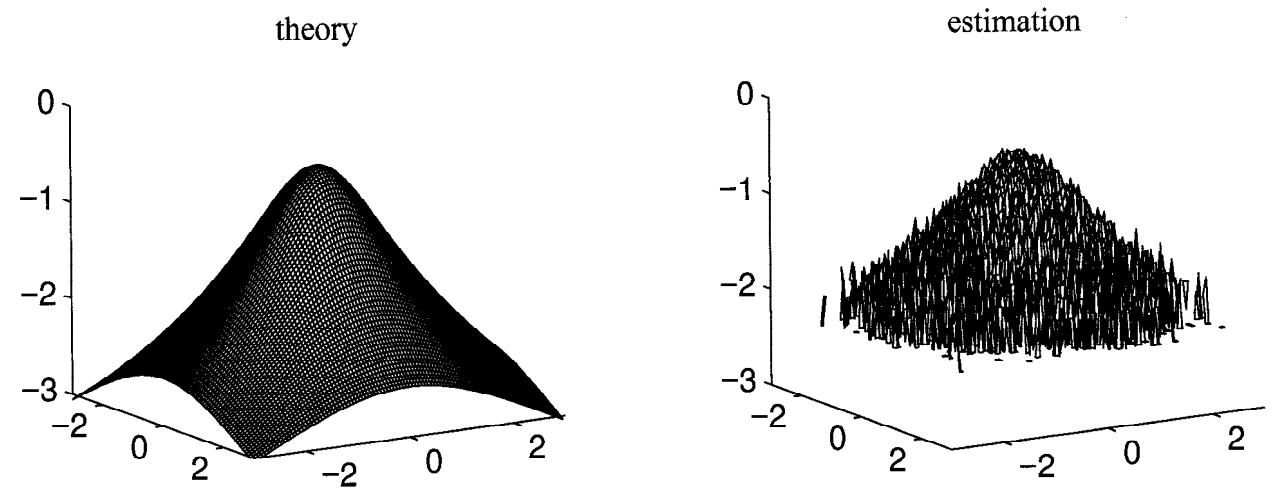

Figure 1. Log-probability density function of reassignment vectors in the Gabor spectrogram case and in a "noise only" situation. Left: theoretical joint density, as predicted by eq.(10). Right: estimated joint density, obtained from a simulation based on an average over 3 noise realizations, each of $412 \times 156$ time-frequency data points.
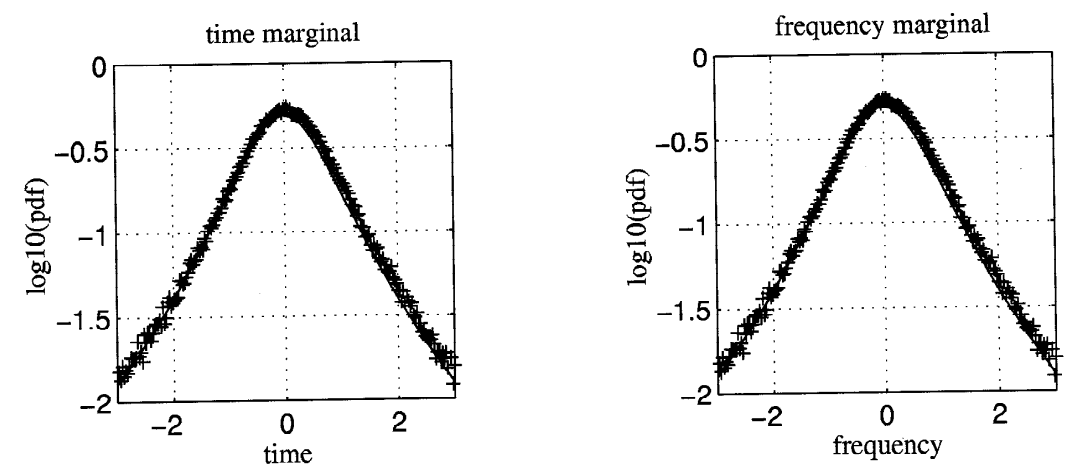

Figure 2. Log-marginals of the probability density function of reassignment vectors in the Gabor spectrogram case and in a "noise only" situation. Left: time marginal, right: frequency marginal. Solid lines correspond to the theoretical predictions of eq.(11), whereas the crosses have been obtained from a simulation based on an average over 3 noise realizations, each of $412 \times 156$ time-frequency data points.

$f_{\underline{F}}\left(F_{1}, F_{2}\right)=\frac{1}{\pi^{2}|\operatorname{det} \boldsymbol{\Gamma}|} \exp \left(-\underline{F}^{\dagger} \boldsymbol{\Gamma}^{-1} \underline{F}\right) ; \quad \boldsymbol{\Gamma}=\left[\begin{array}{cc}\sigma_{1}^{2}=2 \sigma^{2} & 0 \\ 0 & \sigma_{2}^{2}=\sigma^{2} \lambda^{2} / 2 \pi\end{array}\right]$.

Making then the change of variables 


$$
\left[\begin{array}{ll}
F_{1} & F_{2}
\end{array}\right] \rightarrow[w r]\left\{\begin{aligned}
w & =F_{1} \\
r & =-(\sqrt{4 \pi} / \lambda) F_{2} / F_{1}
\end{aligned}\right.
$$

whose inverse transform has for Jacobian $|\lambda w|^{2} / 4 \pi$, we get for the pdf of $r(t, \nu)$ (see [4] for the principle of the technique and [2] for the details):

$$
\begin{aligned}
f(r) & =\frac{\lambda^{2}}{4 \pi} \iint_{-\infty}^{+\infty}|w|^{2} f_{\underline{F}}\left(w,-\frac{\lambda}{\sqrt{4 \pi}} r w\right) d \operatorname{Re}\{w\} d \operatorname{Im}\{w\} \\
& =\frac{\lambda^{2}}{4 \pi^{2}\left(\sigma_{1}^{2} \sigma_{2}^{2}\right)\left[1 / \sigma_{1}^{2}+|\lambda r|^{2} /\left(4 \pi \sigma_{2}^{2}\right)\right]^{2}},
\end{aligned}
$$

a result which - using the definitions of $\sigma_{1}$ and $\sigma_{2}$ - can be more simply reexpressed as

$$
f(r)=\frac{1}{\pi\left(1+|r|^{2}\right)^{2}} \text {. }
$$

As a consequence, $r(t, \nu)$ appears as being zero-mean but of infinite variance, with marginal densities given by (let us recall that the real and imaginary parts of $r$ correspond to the reassignment components in time and frequency, respectively)

$$
f(\operatorname{Re}\{r\})=\frac{1}{2\left(1+(\operatorname{Re}\{r\})^{2}\right)^{3 / 2}} ; \quad f(\operatorname{Im}\{r\})=\frac{1}{2\left(1+(\operatorname{Im}\{r\})^{2}\right)^{3 / 2}} .
$$

It turns out that both quantities have exactly the same form, what results from the radial symmetry of (10). We can furthermore remark that they are independent of the noise level $\sigma^{2}$, of time (stationarity), of frequency (whiteness) and of the window length (reduced coordinates).

Another way of formulating the same results is to make use of the polar coordinates $\rho=|r| \in[0,+\infty[$ and $\theta=\arg r \in]-\pi, \pi]$, thus leading to a joint pdf

$$
f(\rho, \theta)=\frac{\rho}{\pi\left(1+\rho^{2}\right)^{2}}
$$

which is now independent of $\theta$, and therefore to the marginal properties

$$
f(\rho)=2 \rho /\left(1+\rho^{2}\right)^{2} ; \quad f(\theta)=1 / 2 \pi .
$$

\subsection{The "signal + noise" case}

The previous analysis can be carried over to "signal + noise" situations where the observation is of the form $x(t)=s(t)+n(t)$, with $s(t)$ a non-random component and $n(t)$ as in (4). According to this model, $x(t)$ is still Gaussian circular, although not zero-mean.

Calculations are in this case more cumbersome than in the "noise only" case but the derivation proceeds along the same lines (details are given in [2]). The final result is

$$
f(r)=\frac{1}{\pi\left(1+|r|^{2}\right)^{2}}\left[1+\frac{S}{2 \sigma^{2}} \frac{\left|1+r r_{0}^{*}\right|^{2}}{1+|r|^{2}}\right] \exp \left\{-\frac{S}{2 \sigma^{2}} \frac{\left|r-r_{0}\right|^{2}}{1+|r|^{2}}\right\}
$$



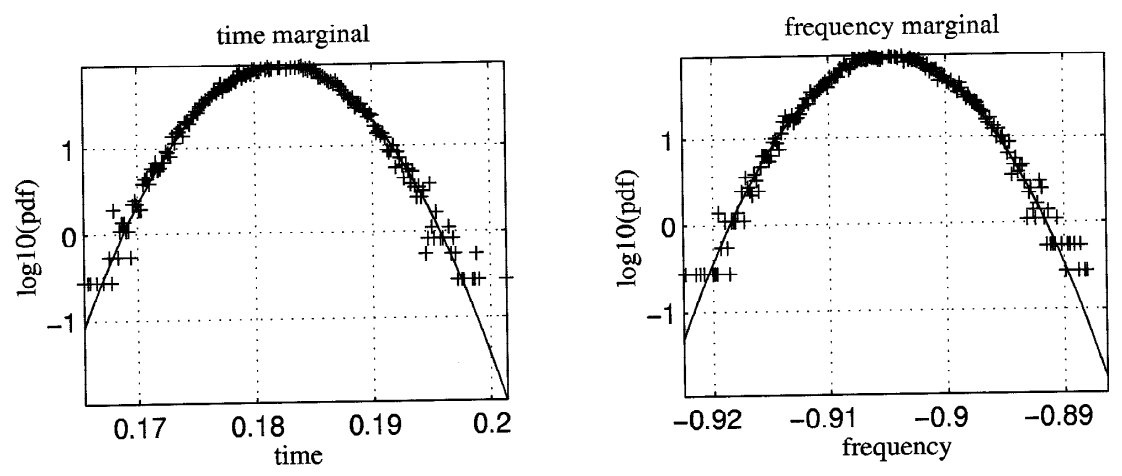

Figure 3. Log-marginals of the probability density function of reassignment vectors in the Gabor spectrogram case and in a "chirp+noise" situation $(\mathrm{SNR}=64 d B)$. Left: time marginal, right: frequency marginal. Solid lines correspond to the theoretical predictions derived from eq.(14), whereas the crosses have been obtained from a simulation based on an ensemble average over 20,000 noise realizations.

with $S=\left|F_{s}^{(h)}(t, \nu)\right|^{2}$ and $r_{0}$ the noise-free reassignment vector.

The pdf now becomes a function of the local signal-to-noise ratio SNR $=S / 2 \sigma^{2}$. In the case where SNR $\rightarrow 0$, one can check that (14) reduces to the "noise only" pdf (10). One can also remark that (14) tends to be approximatively Gaussian for small $r$ 's and, if one has furthermore SNR $\rightarrow+\infty$ ("signal only" case), one gets $f(r) \sim \operatorname{SNR} \exp (-\mathrm{SNR}) \sim 0$ unless $r=r_{0}$, in which case $f(r) \sim \mathrm{SNR} \sim+\infty$.

The theoretical results of this Section are well-supported by numerical simulations, as illustrated in Figures 1 to 3.

\section{Extension to spectrograms with arbitrary windows}

The use of Gaussian windows only may appear as a restriction and it is desirable to consider more general situations. Dealing with arbitrary windows requires however a specific treatment which cannot take advantage of the simplifications which occurred in the Gaussian case. In fact, it turns out that the problem of evaluating the pdf of a reassignment vector based on an arbitrary window can be embedded in a more general problem which can be stated as follows: given an observation $x(t)=s(t)+n(t)$, where $s(t)$ is non-random and $n(t)$ as in (4), what is the joint pdf of the $(N-1)$ dimensional complex-valued vector $\underline{r}=\left[r_{1} r_{2} . . r_{N-1}\right]^{t}$ whose coordinates are given by $\left(r_{n}=\left(1 / \lambda_{n}\right) y_{n+1} / y_{1} ; n=1 \ldots N-1\right)$, where the $\lambda_{n}$ 's are complex-valued numbers and the collection of $y_{n}$ 's results from the linear filtering of $x(t)$ by a family of filters of impulse responses $h_{n}(t)$ ?

This problem can be solved by introducing the change of variables 


$$
\left[y_{1} y_{2} \ldots y_{N}\right] \longrightarrow\left[y_{1} r_{1} \ldots r_{N-1}\right]
$$

whose inverse transform has for Jacobian $\left|r_{1}^{N-1} \prod_{n=1}^{N-1} \lambda_{n}\right|^{2}$. It follows that

$$
f(\underline{r})=\left|\prod_{n=1}^{N-1} \lambda_{n}\right|^{2} \iint_{-\infty}^{+\infty}\left|y_{1}\right|^{2(N-1)} f_{\underline{Y}}\left(\underline{r}_{\lambda} y_{1}\right) d \operatorname{Re}\left\{y_{1}\right\} d \operatorname{Im}\left\{y_{1}\right\},
$$

with $\underline{r}_{\lambda}=\left[\begin{array}{llll}1 & \lambda_{1} r_{1} & \lambda_{2} r_{2} \ldots \lambda_{N-1} r_{N-1}\end{array}\right]^{t}$ and where $f_{\underline{Y}}($.$) stands for the joint density of$ the filtered observations $\left(y_{1}, y_{2}, \ldots y_{N}\right)$, a Gaussian pdf of covariance matrix $\boldsymbol{\Gamma}$.

This expression can be evaluated in a more explicit form and, after some tedious manipulations (for details, see [2]), we get

$$
\begin{aligned}
f(\underline{r})=\frac{1}{\pi^{N}|\operatorname{det} \boldsymbol{\Gamma}|} \mid & \left.\prod_{n=1}^{N-1} \lambda_{n}\right|^{2} e^{-|b|^{2} / a+c} \\
& \iint_{-\infty}^{+\infty}\left|y_{1}+\frac{b}{a}\right|^{2(N-1)} \exp \left(-a\left|y_{1}\right|^{2}\right) d \operatorname{Re}\left\{y_{1}\right\} d \operatorname{Im}\left\{y_{1}\right\},
\end{aligned}
$$

with

$$
a=\underline{r}_{\lambda}^{\dagger} \boldsymbol{\Gamma}^{-1} \underline{r}_{\lambda} ; \quad b=\underline{s}^{\dagger} \boldsymbol{\Gamma}^{-1} \underline{r}_{\lambda} ; \quad c=\underline{s}^{\dagger} \boldsymbol{\Gamma}^{-1} \underline{s}
$$

and $\underline{s}$ the vector formed by the filtered versions of the noise-free observation.

In the "noise only" case where $s(t)=0$, some simplifications occur, leading to

$f(\underline{r})=\frac{1}{\pi^{N}|\operatorname{det} \boldsymbol{\Gamma}|}\left|\prod_{n=1}^{N-1} \lambda_{n}\right|^{2} \iint_{-\infty}^{+\infty}\left|y_{1}\right|^{2(N-1)} \exp \left(-a\left|y_{1}\right|^{2}\right) d \operatorname{Re}\left\{y_{1}\right\} d \operatorname{Im}\left\{y_{1}\right\}$

and, finally, to

$$
f(\underline{r})=\frac{1}{\pi^{N}|\operatorname{det} \boldsymbol{\Gamma}|}\left|\prod_{n=1}^{N-1} \lambda_{n}\right|^{2} \frac{(N-1) !}{a^{N}} .
$$

This result is quite general and can be applied to the specific situation of time-frequency reassignment by setting

$h_{1}(t)=h^{*}(t) e^{i 2 \pi \nu t} ; \quad h_{2}(t)=\left(\mathcal{T} h^{*}\right)(t) e^{i 2 \pi \nu t} ; \quad h_{3}(t)=\left(\mathcal{D} h^{*}\right)(t) e^{i 2 \pi \nu t}$,

thus leading to

$$
\boldsymbol{\Gamma}=2 \sigma^{2}\left[\begin{array}{ccc}
1 & 0 & 0 \\
0 & \Delta t_{h}^{2} & -1 / 2 \\
0 & -1 / 2 & 4 \pi^{2} \Delta \nu_{h}^{2}
\end{array}\right]
$$

It has to be observed that $\operatorname{det} \boldsymbol{\Gamma}=2 \sigma^{6}\left(16 \pi^{2} \Delta t_{h}^{2} \Delta \nu_{h}^{2}-1\right)$, a quantity which, by virtue of Heisenberg's inequality, is always non-negative and is zero if and only if the window is Gaussian. In this case, $\Gamma$ is no longer invertible and it is necessary to go back to the results of the previous Section. 
Assuming that $h(t)$ is not Gaussian, we have to consider the reassignment vector

$$
r=-\frac{1}{\Delta t_{h}} \operatorname{Re}\left\{\frac{y_{2}}{y_{1}}\right\}+\imath \frac{1}{2 \pi \Delta \nu_{h}} \operatorname{Im}\left\{\frac{y_{3}}{y_{1}}\right\}
$$

thus motivating the introduction of

$$
\underline{r}=\left[r_{1} r_{2}\right]^{t}=\left[\frac{1}{\lambda_{1}} \frac{y_{2}}{y_{1}} \frac{1}{\lambda_{2}} \frac{y_{3}}{y_{1}}\right]^{t},
$$

with $\lambda_{1}=-\Delta t_{h}$ and $\lambda_{2}=2 \pi \Delta \nu_{h}$. The joint density of this vector can be shown to take on the form:

$$
f(\underline{r})=\frac{2}{\pi^{2}} \frac{H^{2}\left(H^{2}-1\right)^{2}}{\left[H^{2}\left(1+\left|r_{1}\right|^{2}+\left|r_{2}\right|^{2}\right)-2 H \operatorname{Re}\left\{r_{1} r_{2}^{*}\right\}-1\right]^{3}},
$$

with $H=4 \pi \Delta t_{h} \Delta \nu_{h}$, whence the final result

$$
f(r)=\iint_{-\infty}^{+\infty} f(\underline{r}) d \operatorname{Im}\left\{r_{1}\right\} d \operatorname{Re}\left\{r_{2}\right\}=\frac{1}{\pi\left(1+|r|^{2}\right)^{2}},
$$

which appears to be identical to the one obtained in the Gaussian case (see eq.(10)). We can remark that the frequency marginal of eq.(26) can be considered as well as the instantaneous frequency pdf of a colored bandpass Gaussian noise, with the consequence that its expression is in agreement with the result obtained in [5] by a different method.

The situation of "signal + noise" is far more complicated in the general case than in the Gaussian one and it will not be followed up here (see [2] for elements).

\section{Conclusion}

Statistical properties of reassignment vectors have been derived in simple, yet realistic and important situations. Given these results, it is now possible to incorporate statistical elements in the reassignment process, to validate its use in noisy situations and to increase the significance of the results it gives. It is also believed that this knowledge should help in extracting useful information directly from the reassignment vector field, in a statistical signal processing perspective. Finally, it is worthwhile to mention that the obtained results should be of help for providing a statistical basis to related techniques such as the "ridge and skeleton" method [6], whose principle has much to share with reassignment.

\section{References}

1. F. Auger and P. Flandrin, "Improving the readability of time-frequency and time-scale representations by the reassignment method," IEEE Trans. on Signal Proc., Vol. SP-43, No. 5, pp. 1068-1089, 1995.

2. E. Chassande-Mottin, F. Auger and P. Flandrin, "Statistique des vecteurs de réallocation du spectrogramme," Rapport Interne 96-01, Labo. de Physique, ENS Lyon, juillet 1996. A .ps file of this report can be obtained at the following address: http: //www . physique . ens-lyon.fr/ts/report/r9601.ps.gz

3. B. Picinbono, Random Signals and Systems, Prentice Hall, Englewood Cliffs, 1993.

4. A. Papoulis, Probability, Random Variables and Stochastic Processes, McGraw-Hill, New York, 1984. 
5. H. Broman, "The instantaneous frequency of a Gaussian signal: the one-dimensional density function," IEEE Trans. on Acoust., Speech and Signal Proc., Vol. ASSP-29, No. 1, pp. 103-111, 1981.

6. P. Guillemain and R. Kronland-Martinet, "Characterization of acoustic signals through continuous linear time-frequency representations," Proc. IEEE, 84 (4), pp. 561-585, 1996. 